\title{
Fatal Cardiac Involvement in Systemic Erythematosus Lupus and Seronegative Antiphospholipid Syndrome: A Case Report
}

\author{
Nicola $S^{1}$, Rolla $G^{1}$, Pizzimenti $S^{2}$, Fornero $\mathbf{M}^{1}$ and Brussino $\mathrm{L}^{1 *}$ \\ ${ }^{1}$ Dipartimento di Scienze Mediche, AO Ordine Mauriziano Umberto I, Italy \\ ${ }^{2}$ Azienda Sanitaria Locale Torino 2, ASL Torino 2 - Pneumology Department, Italy \\ *Corresponding author: Brussino L, Dipartimento di Scienze Mediche, AO Ordine Mauriziano Umberto I, Turin, Italy
}

\section{ARTICLE INFO}

Received: 彗 April 09, 2019

Published: 蔧 April 18, 2019

Citation: Nicola S, Rolla G, Pizzimenti S, Fornero M, Brussino L. Fatal Cardiac Involvement in Systemic Erythematosus Lupus and Seronegative Antiphospholipid Syndrome: A Case Report. Biomed J Sci \& Tech Res 17(2)-2019. BJSTR. MS.ID.002960.

\section{ABSTRACT}

Systemic Lupus Erythematosus (SLE) is a chronic connective tissue disorder, found to be associated in up to $40 \%$ of cases to a secondary Antiphospholipid Syndrome (APS). SLE has a wide spectrum of clinical manifestations, ranging from mild to potentially lifethreating conditions. Patients presenting hematologic, renal, central nervous system or cardiac manifestations have been described as having a worse prognosis. Although SLE cardiac involvement alone is rarely responsible for patients' death, the mortality risk significantly increases when an APS is associated, making myocardial infarction and pulmonary embolism the main causes of morbidity and mortality in this group of patients. Here, we report on a case of fatal cardiac involvement in a male patient with a long-standing SLE history, whose post-mortem revealed a pulmonary thromboembolism likely due to a seronegative antiphospholipid syndrome.
\end{abstract}

Abbreviations: SLE: Systemic Lupus Erythematosus; APS: Antiphospholipid Syndrome; DVT: Deep Venous Thrombosis; ANA: Anti-Nuclear Antibodies; ENA: Extractable Nuclear Antigen; ESR: Erythrocyte Sedimentation Rate; CRP: C-Reactive Protein

\section{Introduction}

Systemic lupus erythematosus (SLE) is a multisystem, autoimmune chronic connective tissue disorder characterized by a broad spectrum of clinical presentations [1] which may be associated to a secondary Antiphospholipid Syndrome (APS) [2]. Although most patients complain skin, musculoskeletal, mild hematologic and serosal involvement [3,4], up to $50 \%$ of patients affected by SLE were diagnosed with heart involvement, making cardiovascular diseases the second main cause of death among lupus patients [3]. This is mainly due to myocardial infarction, congestive heart failure and arrythmias, whereas pulmonary embolism and coronaries' vasculitis are very rare [3]. Here, we report on a case of fatal cardiac involvement in a male patient with a long-standing SLE history.

\section{Case Presentation}

A 34-years old non-smoker male presented in December 2013 to the Immunology Outpatients' clinic at Mauriziano Hospital, in Turin, Italy, complaining recurrent chest infections, diffuse arthralgia, weakness and fever over the last three months. The patient's previous medical history revealed a remote anaphylaxis after oral penicillin administration and a transient loss of consciousness a few minutes after taking ibuprofen due to headache when he was 18 . He did not have any positive familiar history of autoimmune diseases, and he was diagnosed with SLE since he was 16 , after a hospital admission due to pericarditis, polyarthritis and fever. Since that, he was successfully treated with prednisone low dose and hydroxychloroquine, that he stopped two years later for wellness. No previous deep venous thrombosis (DVT), embolism or cardiovascular disease were remarked.

Ten years after SLE diagnosis, the patient was again admitted to an Emergency Department complaining intense fatigue and weakness, high fever, pericardial effusion, diffuse lymphadenopathy and weightloss. The suspicious oflymphoproliferative hematological disease was raised, also supported by the finding of hemolytic anemia, and the patient underwent a bone marrow biopsy, negative for malignancy, and a surgical right axillary lymphadenectomy, 
whose results reported a reactive lymphadenopathy with follicular hyperplastic pattern and sinuses histiocytosis, without any evidence of lymphoma. Flow cytometry and marker results did not support malignancy, and acid-fast stain, bacterial, mycobacterial and fungal cultures were all reported as negative. A cardiac ultrasound scan confirmed an anterior $2 \mathrm{~mm}$ pericardial effusion and a thickened and hyperreflective posterolateral pericardium.

Therefore, a three-days course of 1000 mg methylprednisolone intravenous treatment was undertaken, followed by oral prednisone at the dose of $1 \mathrm{mg} / \mathrm{kg}$ tapered to $7,5 \mathrm{mg} /$ day in 12 weeks. Complete relief of fatigue symptoms was obtained, as well as fever remission and a progressive normalization of blood tests. As a maintenance therapy, methotrexate was added on, but unfortunately it had to be stopped after 3 months due to vomiting, diarrhea and liver abnormalities. After five years of complete well-being, the patient was admitted to our Immunology unit as a matter of urgency complaining weakness, sudden dyspnea and chest pain. Blood tests revealed a relapse of the hemolytic anemia, whereas a chest CT scan confirmed a pleuro-pericarditis and a diffuse lymph-nodes enlargement. After ruling out any infectious and hematological reasons, he was diagnosed with a new SLE relapse, and he was treated with cyclophosphamide $1000 \mathrm{mg}$ for three administrations, after that he was lost to follow-up.

On physical examination he was febrile, being his temperature $38.6^{\circ} \mathrm{C}, \mathrm{BP} 100 / 60 \mathrm{mmHg}$ and pale. His chest auscultation revealed crackles on the basis and some rhonchi. He had multiple swollen joints, including both wrists and ankles, as well as the II and III MCP on the right hand, and there was splenomegaly, without any ankle edema, skin rash or nodular lesions. Blood tests revealed an increase in inflammation markers (CRP $25 \mathrm{mg} / \mathrm{dl}$, ESR $83 \mathrm{~mm} / \mathrm{h}$, Fibrinogen $780 \mathrm{mg} / \mathrm{dl}$ ) and a SLE in active phase (ANA 1:1.280 homogenous, C3 $38 \mathrm{mg} / \mathrm{dl}, \mathrm{C} 48,4 \mathrm{mg} / \mathrm{dl}, \mathrm{Ab}$ anti-DNA $52 \mathrm{U} / \mathrm{ml}$, Ab Anti-Sm $37 \mathrm{U} / \mathrm{ml}$ ), which was also confirmed by hemolytic anemia and lymphocytopenia (Hb 8,4 g/dl, MCV 96 fl, LDH 960 U/L, haptoglobin $<0.4 \mathrm{mg} / \mathrm{dl}, \mathrm{WBC} 3800$ cell/mcl, Lymphocytes $900 \mathrm{cell} / \mathrm{mcl}$, Platelets $480.000 \mathrm{cell} / \mathrm{mcl}$ ), whereas both lipid and glycemic profiles were normal. No signs of pneumonia or renal involvement were noticed, and infectious diseases were ruled out, being negative tuberculosis, HIV, HBV, HCV and Parvovirus B19 results.

Due to the recurrent chest infections, the patient was treated with oral prednisone $25 \mathrm{mg}$ and antibiotics (levofloxacin and ceftriaxone) and he was given an appointment for belimumab infusion.

\section{Outcome}

Three days after the outpatients' visit, the patient was again admitted to the Emergency Department complaining sudden chest pain, dyspnea, hemoptysis and persistent fever. On physical examination, he was febrile and sweaty, his pulse was thready and tachy and his 02-sats was dropping (84\% on breathing air). Blood tests revealed a positive D-dimer, an ECG showed a sinus tachycardia with right axis deviation and right ventricular strain pattern, and the chest TC with angiogram confirmed the pulmonary thromboembolism's suspicion. After few minutes, the patients passed out and even though the prompt resuscitation approaches, he died .A postmortem revealed a complete occlusion of the main pulmonary artery, reported as primary cause of death, and showed a large transmural infarction due to wide atherosclerotic plaques in both left and anterior descending coronary arteries.

\section{Discussion}

Systemic lupus erythematosus is a chronic autoimmune disease, in which genetic and environmental factors, as well as innate and acquired immunity are thought to play an important pathogenetic role [1]. The prevalence of lupus is higher in blacks than in Northern European or Americans, varying from 40 to 200 cases per 100.000 inhabitants and the disease occurs more often between the ages of 15 and 44 [5]. SLE is nine times more frequent in women than in men, suggesting a hormones involvement in the ones, although some studies assumed the protective role of male hormones [6]. SLE is characterized by multisystem microvascular inflammation with the generation of many autoantibodies, particularly Anti-Nuclear Antibodies (ANA), Extractable Nuclear Antigen (ENA), including Ro, La, Sm, RNP, Scl-70 and Jo1, Antiphospholipid Antibodies (lupus anticoagulant, anticardiolipin and anti-beta2-glycoprotein I). C3 and $\mathrm{C} 4$ or CH50 complement levels could be reduced, whereas erythrocyte sedimentation rate (ESR) and/or C-reactive protein (CRP) levels could be found increased. A complete blood count might also reveal leukopenia, thrombocytopenia, and/or anemia, maybe associated to multiple iron and vitamin deficiencies [7]. In case of renal involvement, serum creatinine and urinalysis with urine sediment could help to make a more accurate diagnosis [8]. The clinical course of lupus is extremely different among patients, floating from periods of remissions to chronic or acute relapses. SLE has a wide spectrum of clinical manifestations, potentially affecting any organ, although most patients complain skin, musculoskeletal, mild hematologic, and serosal involvement $[2,6]$.

Nevertheless, patients presenting predominately severe hematologic, renal, central nervous system or cardiac manifestations have a worse prognosis [5]. Heart involvement in SLE includes pericarditis, myocarditis, nonbacterial verrucous endocarditis, coronary artery disease, premature coronary atherosclerosis, congestive heart failure, cardiac arrhythmias, pulmonary hypertension, and conduction disturbances, and although the incidence of cardiac involvement at postmortem is approximately fifty percent, less than $1 \%$ of suspected cardiac deaths is definitely attributable to cardiac involvement [9]. Conversely, myocardial infarction, pulmonary embolism and stroke are the main causes of morbidity and mortality in APS, which is known to be associated to SLE in up to $40 \%$ of cases [2]. In the case we reported, the antiphospholipid antibodies were negative, but recently the diagnosis of seronegative APS has been suggested for patients with clinical manifestations indicative of APS and negative results in the commonly used APL assays [2]. To overcome this problem, new emerging laboratory tests have been identified, but their clinical relevance is still debatable and needs to be confirmed by interlaboratory efforts toward standardizing diagnostic tools. 


\section{Conclusion}

Cardiac complications in SLE, reported to be present in up to $50 \%$, are associated to high morbidity and mortality. SLE disease activity, chronic inflammation, genetic predisposition and treatment adverse effects are thought to be involved in the pathogenesis of cardiac damage, mainly if the disease is associated to APS. Nevertheless, a proper diagnosis is not always possible, leading to a dramatical poor prognosis.

\section{References}

1. M Di Battista, Marcucci E, Elefante E, Tripoli A, Governato G, et al. (2018) One year in review 2018: systemic lupus erythematosus. Clinical and Experimental Rheumatology 36(5): 763-777.

2. Hughes GR, Khamashta M (2019) Seronegative antiphospholipid syndrome: an update. Lupus 28(3): 273-274.

3. A Doria, Iaccarino L, Sarzi Puttini P, Atzeni F, Turriel M, et al. (2005) Cardiac involvement in systemic lupus erythematosus. Lupus 14(9) 683-686.

ISSN: 2574-1241

DOI: 10.26717/BJSTR.2019.17.002960

Brussino L. Biomed J Sci \& Tech Res

This work is licensed under Creative Commons Attribution 4.0 License

Submission Link: https://biomedres.us/submit-manuscript.php
4. Bucca C, Brussino L, Maule MM, Baldi I, Guida G, et al. (2011) Clinical and functional prediction of moderate to severe obstructive sleep apnoea. Clinical Respiratory Journal 5(4): 219-226.

5. Johnson AE, Gordon C, Palmer RG, Bacon PA (1995) The prevalence and incidence of systemic lupus erythematosus in Birmingham, England: relationship to ethnicity and country of birth. Arthritis Rheum 38(4): 551-558.

6. Anisur Rahman, David A Isenberg (2008) Systemic Lupus Erythematosus. N Engl J Med 358(9): 929-939.

7. Bucca C, Culla B, Brussino L, Ricciardolo FL, Cicolin A, et al. (2012) Effect of iron supplementation in women with chronic cough and iron deficiency. International Journal of Clinical Practice 66(11): 1095-1100.

8. Daniel J Wallace (2019) Diagnosis and differential diagnosis of systemic lupus erythematosus in adults. UpToDate.com, Literature review current through.

9. Busteed S, Sparrow P, Molloy C, Molloy MG (2004) Myocarditis as a prognostic indicator in systemic lupus erythematosus. Postgraduate Medical Journal 80(944): 366-367. 